\title{
Cognitive systems and operations research in big data and cloud computing
}

\author{
Marek R. Ogiela ${ }^{1} \cdot$ Hoon Ko ${ }^{2}$
}

Published online: 19 April 2018

(c) Springer Science+Business Media, LLC, part of Springer Nature 2018

In recent years, all fields of scientific research related to intelligent information processing, knowledge extraction, optimization, and semantic evaluation have developed extensively. They are not only associated with traditional approaches in which data are acquired and processed, such as computer and management sciences, pervasive computing etc., but also they have become the basis for new scientific disciplines and research areas, such as cognitive informatics, bio-inspired computing, and even some innovative research fields of operations research. In such promising disciplines, which will be based on future generation computing technologies, there will be opportunities to lead scientific research on collecting and analyzing great amount of information, signals, or patterns. Further, understanding the mechanisms by which humans absorb information, semantic meaning, and optimally applying it to learn and solve very complex optimization problems, using artificial thinking processes, and information fusion approaches will be needed. Of course such complex problems may be connected with many interdisciplinary scientific areas, and sometimes may be solved using traditional operations research methodologies; but the increasing complexity of problems, and the availability of larger repositories of information and data, which require intelligent processing and interpretation, will require the application of some new and innovative computation paradigms to find solutions which may be especially connected with big data repositories or cloud computing.

Obviously techniques that can support big data analysis and cloud computing must also meet important requirements for efficient and optimal operation, guaranteeing not only opti-

Marek R. Ogiela

mogiela@agh.edu.pl

Hoon Ko

dr.hoonko@gmail.com

1 Cryptography and Cognitive Informatics Research Group, AGH University of Science and Technology, 30 Mickiewicza Ave, 30-059 Krakow, Poland

2 IT Research Institute, Chosun University, 309, Pilmun-daero, Dong-gu, Gwangju 61452, Republic of Korea 
mal solutions, but also secure transmission and personalized application. Innovative solutions in cognitive informatics, and operations research techniques, can ensure such difficult-toachieve features. This leads to a huge demand for new and effective solutions for intelligent big data processing, having the form of different signals, collected on distributed computers, and used to provide different services via cloud computing infrastructures.

To consider these interesting and significant topics related to the quickly developing field of cognitive systems and operations research, we have edited this special issue, Cognitive Systems and Operations Research in Big Data and Cloud Computing, selecting eight submissions that present the most interesting research on advanced solutions for big data and cloud computing applications.

The paper "Indoor localization and navigation using smartphone sensory data," by HuiHuang Hsu, Jung-Kuei Chang, Wei-Jan Peng, Timothy K. Shih, Tun-Wen Pai, and Ka Lok Man presents an innovative application for indoor localization and navigation using smartphone sensory data. A Pedestrian Dead Reckoning-based approach is presented. Their new system can in real-time track the user's position and movement. The authors propose to use special calibration marks in the form of arrows placed on both the floor plan and the ground, which after calibration show the right direction on the floor plan displayed on a smartphone. When the user starts to move, the system estimates step length and moving direction considering data from an accelerometer and a gyroscope. This application allows one to solve an optimal path planning task. The experimental results show that this approach is practical and feasible, and allows traces with high accuracy.

"Optimal column subset selection for image classification by genetic algorithms," by Pavel Krömer, Jan Platoš, Jana Nowaková, and Václav Snášel, presents a novel variant of genetic algorithms for the column subset selection problem. The described approach employs a recent genetic algorithm for fixed length subset selection, and extends it by a novel crossover operator based on the Mergesort algorithm. Performed experiments on image data showed that the new crossover operator leads to equal or significantly better results than does the basic genetic algorithm. Evaluation of the datasets shows that the classification of the reduced datasets can be efficiently performed by several classification methods, including CART, linear SVM, and linear regression. Other classification algorithms, such as the Naïve Bayes classifier, were not successful in learning the classification of the reduced datasets. Presented results indicate that nature-inspired metaheuristics are promising tools that can be used to find efficient solutions to hard combinatorial optimization problems.

"Human actions recognition from motion capture recordings using signal resampling and pattern recognition methods," by Tomasz Hachaj, Marek R. Ogiela, and Katarzyna Koptyra, presents a new classifier for human motion analysis. The authors prove that after recalculating the motion capture data to position-invariant representation, it can be directly used by a classifier to successfully recognize various actions types. The proposed classifier can deal with objects that are described by a great number of numeric values, and it was applied to perform a classification task on gym exercises and karate techniques datasets. Depending on the dataset, the authors obtained average recognition rates from 100 to 97\%; and found that the proposed features selection and classification approach guarantees high efficiency and effectiveness.

"Experimental comparisons with respect to the usage of the promising relations in EDAbased causal discovery," by Song Ko, Hyunki Lim, Hoon Ko, and Dae-Won Kim, presents some improvements in creating causal relations between nodes in datasets. One of the major problems in a Bayesian network is connected with the creation of causal relations from a dataset by constructing better heuristic learning approaches. Many kinds of approaches are known, and estimation of distribution algorithms (EDAs) is a promising solution. Because the 
performance of EDAs is dependent on the quality of the first selected population, the authors introduce a new initialization method that extracts promising candidate causal relations based on causal scores. Next they use the promising relations to construct a better first population and to reproduce better individuals until the learning algorithm is terminated. Experimental results show that EDAs infer more correct causal relations when promising relations were used in EDA based structure learning.

"A service model for nutrition supplement prediction based on Fuzzy Bayes model using big data in livestock," by Saraswathi Sivamani, Jongsun Choi, and Yongyun Cho, presents a novel method in the decision support system for the nutritional management of livestock using the Bayesian model based on fuzzy rules. This system allows the analysis of information from a Big Data infrastructure, and uses Bayesian logic with the fuzzy linguistic variables, focused on the probabilities of the food intake with respect to some defined fuzzy rules describing predicted values. The fuzzy logic technique helps to decide on the decision system, when there are more than one dependencies; and is applied to determine the health status of the cow, and to predict the nutritional intake in the livestock.

"Including cognitive aspects in multiple criteria decision analysis," by João Carneiro, Luís Conceição, Diogo Martinho, Goreti Marreiros, and Paulo Novais, presents a new multiple criteria decision analysis (MCDA) method, which includes cognitive aspects called Cognitive Analytic Process. This new approach allows decision-makers to configure a multicriteria problem (criteria and alternatives) to include the expertise levels, credibilities, and behavior styles of the decision makers. The authors evaluated the Cognitive Analytic Process in terms of configuration costs and the capability to enhance the quality of the decision. They used the satisfaction level as a metric to compare the presented method with other known MCDA methods. Finally this new solution achieved higher satisfaction levels than other methods, especially when the decision suggested by Cognitive Analytic Process was different from the one proposed by those methods.

"New paradigms of cognitive management extending computational intelligence approaches," by Lidia Ogiela and Urszula Ogiela, presents a new computational paradigm called cognitive management. This approach is especially dedicated to support information management processes that are based on semantic evaluation and extraction of the meaning of the analysed data or information. The management processes supported by this new approach can be performed in different areas and show the versatility of this paradigm. Cognitive management processes are defined with respect to the methods used in the cognitive interpretation of data records and applied for semantic meaning evaluation and for the purpose of supporting existing and traditional data management solutions. Cognitive management processes are discussed as they are applied to various subjects, i.e., data analysis, information fusion and management, knowledge extraction, and secure information distribution in different economical structures.

"Improved performance optimization for massive small files in cloud computing environment," by Chang Choi, Chulwoong Choi, Junho Choi, and Pankoo Kim, presents a new approach to optimize small files storage in a cloud environment. Hadoop uses the Hadoop distributed file system for storing big data, and uses MapReduce to process big data in cloud computing environments. Because Hadoop is optimized for large file sizes, it has difficulties processing large numbers of small files. To overcome this limitation, this study proposes a performance improvement method for MapReduce processing of big data in cloud computing environments that integrates the CombineFileInputFormat method and the reuse feature of the Java Virtual Machine (JVM). Traditional methods create a mapper for every small file, but the proposed approach reduces the number of created mappers by processing large numbers of files that are combined by a single split using CombineFileInputFormat. Additionally, to 
improve MapReduce processing performance, the proposed method reduces creation time by reusing a single JVM to run multiple mappers (rather than creating a JVM for every mapper). The proposed method requires less processing time for large data repositories.

We believe that the papers included in this special issue will facilitate scientific work and contribute to the studies conducted by other industry practitioners and scientific researchers in the area of cognitive systems and operations research dedicated to cloud and big data computing.

We would like to express our sincere appreciation of the valuable contributions made by the authors. Our special thanks go to Prof. Dr. Endre Boros from Rutgers University, Editor in Chief of the Annals of Operations Research, for allowing us to organize and publish this special issue. We would like also to thank to Katie D'Agosta, Publications Manager, for her great support throughout the entire publication process. 